\title{
« Ceux qui font tomber la barrière de couleur au pays de l'apartheid » Le pasteur Georges Mabille et les prémices de la ségrégation raciale d'État en Afrique du Sud
}

\author{
Gilles Teulié, Aix Marseille Univ, LERMA, Aix-en-Provence, France
}

Cet article examine l'Afrique du Sud pendant les premières années de l'apartheid au travers du regard de George Mabille, pasteur-missionnaire de la Société des missions évangéliques de Paris, en poste à Johannesburg de 1946 à 1957 auprès de la communauté des mineurs sotho, une extension de la mission protestante française au Lesotho. Georges Mabille a combattu la ségrégation raciale en Afrique du Sud avec d'autres hommes d'Églises anglophones, alors que le système d'apartheid en était à ses balbutiements, période souvent négligée par les chercheurs. Gilles TEULIÉ s'appuie sur le concept de microhistoire telle que l'a définie Carlo Ginzburg, ainsi que, en ce qui concerne le corpus, sur les archives de Georges Mabille conservées au Défap et les témoignages de personnes l'ayant bien connu, afin de rendre compte de la façon dont la résistance à l'apartheid a été initiée sur place, sous quelles formes, dans quel contexte et pour quelles conséquences.

Il s'agissait d'un appel pressant pour que je revienne en Afrique du Sud pour occuper le poste de missionnaire de Johannesburg au Transvaal. Depuis plusieurs mois une rébellion mettait en danger l'existence même des Églises du Rand et toute l'œuvre missionnaire entreprise parmi les Basotho. Le missionnaire en charge du district avait été amené à quitter son poste, sous la pression des événements ${ }^{1}$.

C'est en ces termes que le pasteur Georges Mabille explique l'urgence avec laquelle la Société des missions évangéliques de Paris souhaite sa présence en terre australe au lendemain de la Seconde Guerre mondiale. Les événements qu'il relate, et qui ont conduit son prédécesseur à quitter précipitamment son poste de Johannesburg, font partie d'une très longue série de conflits opposant, à toutes les époques de l'histoire de l'Afrique du Sud, Blancs et peuples autochtones africains, depuis l'arrivée des premiers Européens qui réussirent à circumnaviguer en passant par le cap de Bonne Espérance au $\mathrm{XV}^{\mathrm{e}}$ siècle jusqu'à l'élection de Nelson Mandela comme président de la République en 1994. Il s'agit donc d'un épisode de plus dans l'histoire de la violence endémique qui secoue le pays et ses habitants depuis des siècles et qui, dans ce cas précis, met en danger l'œuvre de la Mission de Paris non seulement dans la cité de l'or qu'est Johannesburg, mais également non loin de là, au Lesotho (ancien Basutoland ${ }^{2}$ )-où, depuis les années 1830, des générations de missionnaires protestants français, les Casalis, Coillard, Mabille et Ellenberger, sont à l'œuvre, parfois en concurrence

\footnotetext{
${ }^{1}$ Georges Mabille, Un Noir + un Blanc = une équipe. Ceux qui font tomber la barrière de couleur au pays de l'apartheid. Témoignage à Michael Malefane par son co-équipier Georges Mabille, publication privée, 1976, p. 69.

2 Terre des Sotho, aussi Basotho ou Bassouto pour le pluriel et mosotho pour un individu, qui parlent le sesotho.
} 
avec leurs compatriotes missionnaires catholiques. En cette année 1946, la Mission de Paris a besoin d'un pasteur expérimenté, qui connaisse bien le pays, n'ait pas peur de vivre dans une situation troublée et ait la capacité de rétablir l'ordre là où le chaos s'est installé. Georges Mabille est le pasteur de la situation. L'historien Frédéric Fabre écrit à son sujet : " Homme d'ordre typiquement protestant - tel François Guizot en son temps - aumônier et missionnaire patriote, il avait eu un rôle de reprise en main sur les populations chrétiennes fragilisées ${ }^{3}$. » $\mathrm{Ce}$ que Georges Mabille et sa famille ne savent pas, c'est qu'ils sont sur le point de vivre l'une des périodes les plus sombres de l'histoire de l'Afrique du Sud : en 1948, soit moins de deux ans après leur installation à Johannesburg, un ancien pasteur de l'Église réformée néerlandaise d'Afrique du Sud ${ }^{4}$, descendant de Huguenots du Lubéron, Daniel François Malan (18741959), devenu homme politique, instaure le système de ségrégation raciale appelé apartheid. Georges Mabille ne peut tolérer ce système : "Cette société est faite d'insécurité, de frustration et de cruauté, à cause de toutes les lois iniques et injustes imposées par les Blancs à ceux qui n'étaient, après tout, que leurs esclaves ${ }^{5}$. » Il décrit le gouvernement de Malan comme «un gouvernement lié mentalement et spirituellement à une tyrannie aussi intransigeante que celle que voulaient les nazis et les fascistes ${ }^{6} \gg$. Georges Mabille et sa famille sont ainsi des témoins privilégiés des débuts de l'apartheid. Ils assistent à sa mise en place progressive, en particulier la promulgation d'un certain nombre de lois ségrégatives comme l'interdiction des mariages mixtes (1949), des relations sexuelles entre groupes ethniques différents (1950) ou la séparation de ces mêmes groupes (1950). Sa foi dicte au pasteur Mabille de ne pas être simplement témoin de l'évolution du pays, mais également acteur, un acteur réformé et informé qui lutte indirectement contre les Églises réformées néerlandaises d'Afrique du Sud. Il entre en résistance, active ou passive, et est l'un des seuls pasteurs français en poste dans le pays, si ce n'est le seul, à lutter au côté de nombreux ecclésiastiques - anglicans, méthodistes, presbytériens, etc. - sud-africains.

Cette étude s'inscrit dans une démarche relevant de la microhistoire, telle que définie par Carlo Ginzburg ${ }^{7}$. Il s'agit d'examiner la dialectique entre le vécu individuel et les forces sociales auxquelles l'individu s'oppose, au travers des traces qu'il laisse. L'historien dispose de pléthore de documents écrits par Georges Mabille : archives que sa famille a léguées au Service protestant de mission à Paris (le Défap ${ }^{8}$ ), mais également ses livres, auxquels on peut ajouter la mémoire de ce que ses enfants ont vécu. Jacques Revel parle de l'histoire « au ras du sol ${ }^{9}$ », c'est-à-dire au plus près des acteurs de cette histoire et non une histoire qui serait élaborée en surplomb, à partir des données chiffrées d'une période. L'approche microhistorique implique que, quel que soit l'aspect singulier de la vie d'une personne, la

\footnotetext{
${ }^{3}$ Frédéric FABRE, Protestantisme et colonisation. L'évolution du discours de la mission protestante française au XX $X^{e}$ siècle, Paris, Karthala, 2011, p. 146.

${ }^{4}$ La Nederduitse Gereformeerde Kerk (NGK) en afrikaans, le néerlandais créolisé des Afrikaners.

${ }^{5}$ G. MABILle, Un Noir + un Blanc = une équipe, op. cit., p. 97.

${ }^{6}$ Georges MABILlE, «Une expérience communautaire en Afrique du Sud », manuscrit, , Fonds Mabille, Défap, 1955 (?), p. 53 (le document commence à la p. 50). Nous ferons toujours suivre la date de publication d'un point d'interrogation entre parenthèses lorsqu'elle est incertaine. Brouillon partiel de ID., Centre œecuménique interracial de Wilgespruit en Afrique du Sud, publication privée, 1977.

${ }^{7}$ Voir Carlo Ginzburg, Le Fromage et les Vers. L'univers d'un meunier frioulan du XVI siècle, Paris, Aubier, 1980. Nous n'évoquerons pas ici la question de la microhistoire globale, dont le but est d'allier la microhistoire développée depuis les années 1970 et l'histoire globale en vogue depuis les années 1990. Voir Romain BERTRAND, Guillaume CALAFAT, «La microhistoire globale. Affaire(s) à suivre », Annales. Histoire, Sciences sociales; 73 (2018), p. 1-18.

${ }^{8}$ Défap est l'acronyme de Département évangélique français d'action apostolique. Le siège de l'organisation, fondée en 1971, est à Paris. Informations en ligne: https://www.defap.fr/dou-vient-defap/ (consulté le 19 décembre 2019).

${ }^{9}$ Jacques REVEL, «L'histoire au ras du sol », préface à Giovanni LEVI, Le pouvoir au village. Histoire d'un exorciste dans le Piémont du XVII e siècle, trad. Monique Aymard, Paris, Gallimard, 1989.
} 
valeur de l'examen que l'on en fait ne réside pas dans son côté unique, mais dans son exemplarité, dans la manière dont les actes et les pensées de cette personne servent de manière allégorique à la compréhension de problématiques plus larges qui affectent la culture sur un plan global ${ }^{10}$. C'est ainsi que Georges Mabille, sa pensée, ses valeurs, sa foi, ses témoignages, la mémoire qui lui est liée, constituent une passionnante approche microhistorique des débuts de l'apartheid ${ }^{11}$. À travers le combat de Georges Mabille contre la discrimination raciale, cet article a pour objectif d'étudier la façon dont la résistance des chrétiens de l'intérieur de l'Afrique du Suda pu saper les certitudes de l'Église d'État (la NGK), et a amené celle-ci à renoncer à la ségrégation, entraînant avec elle toute la communauté blanche pro-apartheid, sous la conduite du président Frederik de Klerk ${ }^{12}-$ un autre descendant de Huguenots -, convaincu de l'impasse dans laquelle se trouvait son pays à la fin des années 1980.

Plusieurs travaux qui traitent du Centre œcuménique de Wilgerspruit, cocréé par Georges Mabille et cinq autres personnes (trois Blancs et deux Noirs), se sont focalisés sur les années les plus dures de l'apartheid (les années 1970 à 1980), en ne manifestant qu'un intérêt relatif pour les débuts du Centre fondé en décembre 1948 (probablement par manque de sources). Or, ces années sont fondamentales pour comprendre le développement de car c'est au cours de celles-ci que la ségrégation officielle se met en place, avec tous ses tâtonnements et ses approximations, tandis que la résistance commence à s'organiser. Outre l'étude des formes de résistance-qui se mettent progressivement en place, cet article vise à appréhender la manière dont on devient activiste politique pour lutter contre l'injustice dans un pays étranger, dans une situation exceptionnelle comme l'apartheid.

\section{GEORGES MABILLE L'AFRICAIN}

Le choix du pasteur Mabille pour le poste de Johannesburg par la Mission de Paris en 1946 s'explique par le fait qu'il a une très bonne connaissance de l'Afrique du Sud. Il naît en effet en 1909 à Morija ${ }^{13}$, au Lesotho. Enclave indépendante au cœur de l'Afrique du Sud à la suite d'un accord signé entre le roi sotho Moshoeshoe $\mathrm{I}^{\mathrm{er}}$ (1800-1870) et les Britanniques, le Lesotho commence par être un protectorat de la Couronne britannique avant d'accéder à l'indépendance en 1966. Georges Mabille est membre d'une famille de missionnaires

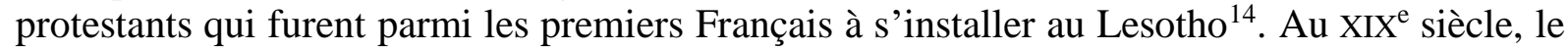
roi Moshoeshoe, en homme avisé, invite des missionnaires protestants français à venir s'installer au Lesotho afin de prodiguer une éducation occidentale à la population. La Mission de Paris envoie Thomas Arbousset (1810-1877), Eugène Casalis (1812-1891) et Constant Gosselin (1800-1872) qui débutent leur mission sur place en juin $1833^{15}$. En 1860, Adolphe

\footnotetext{
${ }^{10}$ Lepore JiLL, « Historians Who Love Too Much. Reflections on Microhistory and Biography », Journal of American History 88 (2001), p. 129-144 (en particulier p. 133).

${ }^{11}$ Cette étude sur le protestant Georges Mabille s'inscrit dans la réflexion menée par l'un des pères fondateurs de la microhistoire, Carlo Ginzburg, en particulier son article sur le calviniste suisse de Neuchâtel Jean-Pierre Purry (1675-1736) qui a voyagé en Afrique du Sud et a porté un regard bienveillant sur les esclaves du Cap alors sous la tutelle de la Compagnie néerlandaise des Indes orientales. Voir Carlo GinZBURG, «Latitude, Slaves, and the Bible. An Experiment in Microhistory », Critical Inquiry 31 (2005), p. 665-683.

12 Leclerc par son père et Foucher par sa mère.

${ }^{13}$ Le nom de la mission de Morija vient de la Bible (Gn 22,2).

${ }^{14}$ Lors d'une visite en mars 2019, Christophe Farnaud, ambassadeur de France en Afrique du Sud et au Lesotho, s'est rendu sur les tombes de la famille Mabille pour saluer la mémoire des premiers Français venus dans le pays.

15 Voir Eugène CASALIS, Les Bassoutos, nouvelle édition présentée et annotée par Jean-François Zorn, Pau, Centre d'étude du protestantisme béarnais, 2012 ; Marie-Claude Mosimann-BARBIER, Un Béarnais en Afrique Australe ou l'extraordinaire destin d'Eugène Casalis, Paris, L'Harmattan, 2012.
} 
Mabille (1836-1894), missionnaire suisse du canton de Vaud, est envoyé par la Mission de Paris au Lesotho auprès du roi Moshoeshoe, avec son épouse Adèle, née Casalis (1840-1923), fille du premier mariage d'Eugène Casalis avec Sarah Dyke (1815-1854). Le fils d'Adolphe et Adèle Mabille, Louis Mabille (1869-1937), épouse Mary Cadier (1875-1974) le 4 janvier 1900. Ils ont de nombreux enfants dont Magali, Henri (Mohato, nom sotho qui signifie « premier pas, première étape »), Hélène, Georges (Tseliso qui veut dire « confort »), Yvonne et Liliane ${ }^{16}$. Henri Mabille (1902-1976) et Georges Mabille (1909-1998) deviennent pasteursmissionnaires et chefs de mission protestante au Lesotho, puis sont incorporés dans les forces françaises. $\mathrm{Au}$ début du $\mathrm{XX}^{\mathrm{e}}$ siècle, les pasteurs-missionnaires de la Mission de Paris ne peuvent rentrer en France que tous les six ans. C'est donc en 1919, au lendemain de la Première Guerre mondiale, que les enfants Mabille découvrent la France. Des années difficiles les attendent, car les parents laissent les quatre aînés en France dans des familles d'accueil afin qu'ils puissent recevoir une bonne éducation, ce qui veut dire qu'ils ne revoient pas leurs parents au cours des années suivantes. Outre le manque affectif, Georges souffre beaucoup de la faim dans cette France d'après-guerre ${ }^{17}$. Ce n'est qu'après avoir été quasiment adopté, par un couple sans enfant - Édouard Jaeglé (1875-1941), pasteur à Colmar de 1901 à 1940, et Jeanne (Scheurer) Jaeglé (1880-1971) ${ }^{18}$-, que Georges peut développer son potentiel. Il obtient son baccalauréat en philosophie à Strasbourg. En 1923, en apprenant le décès de sa grand-mère, Adèle Mabille - ce jour-là, il court derrière un arbre pour cacher sa peine -, il décide de devenir missionnaire, ainsi que le rapporte sa fille Claire. Georges reste fidèle à cette décision et entreprend des études de théologie réformée à la Faculté de Montpellier, sur les recommandations de la Mission de Paris, comme l'atteste une lettre de Paul Barnaud, son directeur-adjoint ${ }^{19}$. Le 30 août 1928, le pasteur Alfred Cadier (1847-1933), père de Mary, épouse de Louis Mabille, écrit d'Osse-en-Aspe au doyen de la Faculté de théologie de Montpellier, Léon Maury (1863-1931), pour lui recommander son petit-fils Georges, afin qu'il puisse succéder à son autre petit-fils Henri (frère de Georges), ainsi qu'à son neveu et filleul Jean Cadier, de même qu'à son neveu Jacques Bost, qui ont tous fait leurs

\footnotetext{
${ }^{16}$ Les membres de la famille Mabille ont tous des noms sotho, insigne honneur donné à des Blancs par les Sotho (Claire s'appelle Tselane, Christian Moshesh, Frank Saiso, Roland Khotso, etc.). Il est remarquable que la mémoire de cette famille de missionnaires ne se soit pas estompée. Lorsque deux des fils de Georges et Louise (Marcelle) Mabille, Christian et Roland, retournent au Lesotho en 2004 pour y enterrer les cendres de leur père selon les dernières volontés de celui-ci, ils font le chemin de leurs aïeuls - une sorte de pèlerinage familial. Lors de leur passage à Wilgespruit, centre multiracial pendant l'apartheid, cofondé par leur père, on leur demande de laisser une partie des cendres de Georges sur place tant son empreinte est encore présente. Mais Christian Mabille refuse, arguant que ce n'était pas la volonté de son père. Une fois au Lesotho, les deux frères sont accueillis avec joie par les Sotho après qu'ils ont décliné leur identité. Roland Mabille raconte que les Sotho affirment à cette occasion que Christian et lui sont chez eux au Lesotho car cet ancrage est attesté par la huitaine de tombes Mabille au cimetière de Morija. Une grande cérémonie d'enterrement des cendres a lieu en présence des autorités religieuses et civiles du Lesotho le 2 février 2004.

17 Claire Casalis raconte à ce propos qu'une dame, amie de la famille, a tellement pitié de lui qu'elle l'emmène dans une pâtisserie et, une fois à l'intérieur, lui dit, à sa grande joie: «Mange tout ce que tu veux. » Entretien avec Claire (Mabille) Casalis, Marseille, 25 juin 2019.

18 «Jaeglé, Frédéric Eugène (1833-1906), son fils Georges et leurs proches », correspondance familiale, compléments historiographiques, biographies, en ligne : http://correspondancefamiliale.ehess.fr/index.php?5237, mis à jour le 17 mai 2019 (consulté le 7 juillet 2019).

${ }_{19}$ Paul Barnaud y précise que la Société a décidé de transformer son école de formation des missionnaires en « une école strictement de technique et d'application missionnaires ». Pour la théologie, la Mission de Paris souhaite désormais confier ses missionnaires aux Facultés de théologie. À cet effet, elle envoie à Montpellier une «solide équipe d'étudiants missionnaires » pour la rentrée 1928, composée d'Édouard Oechsner de Coninck (1909-2004), qui ira par la suite au Cameroun et à Madagascar, Georges Mabille et Jean Nouvelon. Nous nous appuyons ici sur une lettre de Paul Barnaud, directeur adjoint, sur papier à en-tête de la SMEP, écrite de Paris le 2 août 1928, adressée à « cher Monsieur et ami » (en toute hypothèse le doyen de la Faculté de théologie de Montpellier Léon Maury ). Dossier d'étudiant de Georges Mabille, Montpellier, Bibliothèque de la Faculté de Montpellier, Institut protestant de théologie.
} 
études à Montpellier. Alfred souligne que son petit-fils Georges est prêt à suivre les cours de la Faculté comme élève missionnaire et insiste sur sa vocation :

Né dans un champ de mission, il entrevoit la vie missionnaire avec l'attrait profond d'une vocation véritable. Son père Louis Mabille, son grand-père paternel Adolphe Mabille, gendre d'Eugène Casalis, le fondateur de la mission du Lessouto [ancienne graphie de l'actuel Lesotho], semblent lui tracer la route qu'il doit suivre à son tour et que suit au Gabon son frère Henri. Du reste, du côté de ma famille, nous pouvons aussi nous réclamer de François Coillard, cousin de mon père, et qui, à ce titre, était venu me voir dans ma paroisse d'Osse. Il le dit luimême, mon petit-fils Georges Mabille : « je désire consacrer ma vie au service de l'Évangile en pays païen $»^{20}$.

Dans sa lettre de motivation, datée du 30 août 1928 à Osse, Georges Mabille déclare qu'il sait que son frère Henri a été apprécié à Montpellier et qu'il espère, par son travail et son activité, être un bon étudiant ${ }^{21}$. La lettre de recommandation du conseil presbytéral de l'Église réformée d'Osse pour l'entrée de Georges à la Faculté de théologie de Montpellier, signée du président du conseil presbytéral, le pasteur Bost, précise que «ce jeune homme, issu d'une lignée de pasteurs et dont parents et grands-parents sont connus et aimés à Osse, s'est fait connaître dans cette paroisse par son amabilité, son sérieux et sa piété2 ». Georges fait donc ses études à Montpellier du 2 novembre 1928 au 30 juin 1934. Au début des années 1930, ses parents, Louis et Mary, sont en poste dans l'Église du Lesotho sur le Rand à Johannesburg. Leur fils Henri (Mohato) vient les rejoindre avec sa famille puis, en septembre 1934, c'est au tour de Georges Mabille et sa famille de passer à Johannesburg avant de se rendre au Lesotho, où Georges est envoyé en poste par la Mission de Paris. Georges Mabille épouse Marcelle (Louise) Coustère (1908-2003) le 23 septembre 1933 ; il soutient par ailleurs sa thèse de licence en théologie le 30 juin 1934, la même année que sa consécration. Intitulée Les écoles d'initiation en Afrique et la Mission, cette thèse témoigne d'une réflexion caractérisée par l'ouverture à l'autre : "Quelle que puisse être l'attitude que nous adoptions, prenons garde de ne pas nous faire les champions de la civilisation en allant prêcher l'Évangile ${ }^{23}$. » Sa consécration (aujourd'hui selon la terminologie de l'Église protestante unie de France : ordination-reconnaissance de ministère) se déroule le 12 août 1934 à Pau : il est entouré de sa famille et de dix-sept pasteurs-consacrants, dont celui qui fait office de père adoptif le pasteur de Colmar Édouard Jaeglé, qui délivre la prédication de reconnaissance sur Rm 15,13. Le pasteur Alfred Casalis salue Georges Mabille au nom de la Société des missions, en lui souhaitant un bon début de ministère au Lesotho comme son arrière-grand-père, son grandpère et son père ${ }^{24}$.

Après avoir retrouvé Louis et Mary Mabille à Johannesburg, Georges et Marcelle Mabille se rendent à Morija, leur destination, à quelque quatre cent-soixante kilomètres, où pendant plusieurs années Georges œuvre pour la Mission. Puis, c'est la guerre. En 1939, Georges Mabille n'a que six jours pour rejoindre le Cap. En 1940, il est prisonnier en Allemagne puis libéré. En 1942, les besoins sont pressants dans les territoires français en matière de cadres

${ }^{20}$ Lettre manuscrite d'Alfred Cadier au doyen de la faculté de théologie de Montpellier, 30 août 1928. Dossier d'étudiant de Georges Mabille, op. cit.

21 Ibid.

${ }^{22}$ Lettre de recommandation du conseil presbytéral de l'Église réformée d'Osse, séance du 14 octobre 1928 , signée du pasteur Bost, président (ibid.). Selon le témoignage de Roland Mabille, fils de Georges, le tableau n'était pas aussi idyllique : son père était perçu dans la famille «comme un petit galopin ».

${ }^{23}$ Georges MABILLE, Les écoles d'initiation en Afrique et la Mission, thèse soutenue à la Faculté libre de théologie protestante de Montpellier pour obtenir le grade de Bachelier en théologie, tapuscrit, non publié, archives privées de la famille Mabille/archives de la Faculté de Montpellier, Institut protestant de théologie.

${ }^{24}$ Article de journal découpé dans Le Christianisme au XX ${ }^{e}$ siècle, p. 456 (date absente), archives privées de la famille Mabille. 
militaires et d'aumôniers en particulier. Il est envoyé en Afrique Occidentale jusqu'en 1944. Il participe ensuite aux combats en Europe avec les troupes coloniales, tout comme son oncle Ernest Mabille qui avait commandé des Sotho en Europe pendant la Première Guerre mondiale. Dans le même temps, sa famille doit rentrer en France. À la fin de la guerre, on le retrouve en Côte d'Ivoire. C'est à la fin de l'année 1945 qu'il reçoit une lettre officielle du président de la Conférence missionnaire au Lesotho lui demandant de prendre en urgence le poste de la mission de Johannesburg car la situation est délicate : «On me priait de me porter au secours, car aucun autre renfort ne s'avérait disponible à ce moment-là. Il était du reste indispensable que l'éventuel candidat au poste eût une connaissance approfondie de la langue et de la mentalité des Basotho ${ }^{25}$. » Arrivé à Johannesburg en octobre 1946, il retrouve Michael Malefane, l'évangéliste sotho formé par son père et qui l'avait porté, enfant, sur ses épaules. Il se rend au Lesotho pour un court séjour et retourne ensuite occuper officiellement son poste à Johannesburg ; celui-ci comprend la gestion de plusieurs paroisses de mineurs sotho employés dans les mines d'or du Witwatersrand (littéralement « la crête des eaux blanches »), dont celle de Village Main.

\section{CHRONIQUE D'UN APARTHEID ORDINAIRE}

C'est en 1947, soit quelques mois avant la mise en place de l'apartheid, que Georges Mabille rencontre Nelson Mandela, alors jeune père de famille et encore étudiant en droit à l'université de Johannesburg, seul Sud-Africain noir dans ce cas et victime de racisme. Nous n'avons pas beaucoup d'informations sur le lien entre les deux hommes. Toutefois, Claire Casalis, sa fille, a l'intime conviction que son père connaissait Mandela. Et, en effet, dans un livret écrit sur son ami et co-fondateur de Wilgespruit, Arthur Blaxall (1891-1970), le pasteur Mabille relate: «En 1947, j'ai rendu visite à Nelson Mandela, jeune homme d'ici, non violent, dans son bureau, et dès le début il fut membre des amis du Centre de Wilgespruit ${ }^{26}$. » Dans une prédication donnée lors d'un culte un 10 juillet à Osse ${ }^{27}$, Georges Mabille parle de Nelson Mandela comme étant «l'un de nos sympathisants ${ }^{28} »$. Cette affirmation témoigne bien du fait que Georges Mabille gravite dans les milieux intellectuels noirs opposés à la ségrégation, probablement dans le réseau des Églises. Confirmation en est également apportée dans le fait que la plupart des membres fondateurs de Wilgespruit connaissent Mandela ; c'est le cas notamment de Jacob Nhlapo, ou encore d'Arthur Blaxall qui lui rend visite à trois reprises alors qu'il est en attente de son procès en 1963. Détail intéressant : en 1947, Nelson Mandela vit avec sa famille à Orlando, banlieue de Johannesburg, dont le pasteur de la Mission de Paris n'est autre que l'ami de Georges Mabille, Michael Malefane ${ }^{29}$. L'Église évangélique du Lesotho sur le Rand, fondée par les missionnaires de la Mission de Paris, est implantée dans les quartiers défavorisés de Johannesburg, dont Village Main, Orlando, Jabavu, etc. Mandela étant chrétien (méthodiste), il n'est pas impossible que leurs chemins se soient croisés à ce moment-là dans les cercles religieux. Mandela est alors encore étudiant en droit, mais il milite déjà au sein de l'African National Congress (ANC) et en particulier à la Ligue de la jeunesse (African National Congress Youth League) dont il est l'un des fondateurs en 1944, et dont il devient le secrétaire en 1947. Très apprécié dans les instances de l'ANC, il

\footnotetext{
${ }^{25}$ G. MABILLE, Un Noir + un Blanc = une équipe, op. cit., p. 69.

26 Georges MabiLle, Arthur Blaxall, victime de l'Apartheid, par Georges Mabille, son co-équipier, publication privée, 1988 (?).

${ }^{27}$ Prédication manuscrite de Georges Mabille, Fonds Mabille, Défap. La prédication est manuscrite, nous n'avons pas la date exacte, mais l'année 1988 est mentionnée dans la prédication.

${ }^{28}$ Prédication manuscrite de Georges Mabille, Fonds Mabille, Défap.

${ }^{29}$ Georges Mabille a rendu hommage à Michael Malefane dans G. MABILLE, Un Noir + un Blanc = une équipe, op. cit.
} 
est ainsi invité en 1947 à rejoindre le comité exécutif de sa branche du Transvaal. La remarque de Georges Mabille selon laquelle Mandela faisait partie des amis du centre de Wilgespruit montre que les fondateurs du centre cherchent des soutiens parmi les intellectuels noirs anti-ségrégation. De même, ces intellectuels, dont Mandela, n'hésitent pas à renforcer leur réseau, en s'associant à divers projets, y compris des projets d'Église. Ces réseaux sont souvent secrets. Roland Mabille se souvient que son père recevait des pasteurs noirs et blancs dans son bureau situé au sous-sol de leur maison. Roland Mabille avait conscience que ce n'était pas tout à fait légal mais ne s'en inquiétait pas; en fait, il ne se rendait pas bien compte de la situation politique du pays et des conséquences des choix de ses parents.

Georges Mabille écrit beaucoup. Il a une analyse fine de la situation en Afrique du Sud, grâce à ses qualités intellectuelles mais également du fait même qu'il est en poste sur place et qu'avec d'autres il a vu monter le Parti national. Il décode bien le fonctionnement du gouvernement d'apartheid, comme le montre un article de lui paru en 1955 dans le journal protestant français Réforme :

Depuis la prise de pouvoir par le gouvernement nationaliste, les changements se font progressivement et sans éclat. Cependant, chaque mois, un nouveau décret ou une loi, inconnus jusque-là font leur apparition; le réseau des règlements et des interdictions se resserre implacablement ${ }^{30}$.

Georges Mabille souligne que le gouvernement mène une politique d'endormissement de son opposition libérale qui se trouve démunie face à cette politique du fait accompli. Il ose une comparaison entre l'Afrique du Sud et l'Allemagne nazie que beaucoup feront par la suite : "C'est une attitude similaire à celle des éléments libéraux d'Allemagne qui favorisa la poigne de fer de Hitler et lui rendit la tâche aisée ${ }^{31}$. » Tout comme beaucoup d'Européens pensaient la Seconde Guerre mondiale improbable à quelques semaines de l'annexion de l'Autriche, un même phénomène de déni se produit selon lui, aussi bien sur place qu'en Europe :

En Afrique du Sud, ce développement de la situation est masqué à la population européenne par le fait qu'il [le libéral] vit et qu'il se berce de l'illusion, qu'un jour, tout finira bien par s'arranger. Le libéral rejette l'Apartheid qu'il considère être comme une théorie impossible à mettre en pratique. Il ne prend pas au sérieux la législation actuelle, sauf quand elle le touche directement. Le libéral considère les critiques de l'étranger, en particulier d'Europe et d'Amérique, comme mal venues et mal informées et il se recroqueville dans sa coquille ${ }^{32}$.

Sans entrer dans les détails historiques, Georges Mabille a bien compris que derrière l'apartheid se cache une soif de revanche de la part des Afrikaners nationalistes qui fait d'eux les militants d'une cause qui ne souffre aucun compromis tant avec les Noirs qu'avec les Britanniques qui leur ont pris leur pays et leur liberté à l'issue de la guerre anglo-boer (18991902) :

Le peuple Afrikaner $[s i c]$ est décidé à reprendre en main le contrôle total des affaires du pays. Il s'est fixé un but, qu'il compte atteindre par paliers successifs. Au fur et à mesure qu'il s'élève d'un palier au palier supérieur, il augmente ses chances de succès et il est toujours plus convaincu d'être dans le vrai, parce que le Dieu de ses pères est à ses côtés ${ }^{33}$.

\footnotetext{
${ }^{30}$ Georges MABILLE, «EN AFRIQUE DU SUD les nationalistes ont les mains libres », Réforme, 8 octobre 1955 , p. 4.

${ }^{31}$ Ibid.

32 Ibid.

${ }^{33} \mathrm{Ibid}$
} 
On peut dire que, très tôt, Georges Mabille a mis les Européens en garde contre ce système inique basé sur une totale méconnaissance de l'autre : "Il n'y a pas un blanc sur dix, au Sud de l'Afrique, qui sache ce que c'est que d'être un noir. La majorité des blancs ne connaît que son domestique, son employé dans l'industrie ou "la menace noire" ${ }^{\text {"4 }}$. » Il souligne que seuls ceux qui ont pour fonction d'être auprès de Noirs les connaissent, à savoir le magistrat des affaires indigènes, l'assistant social ou le missionnaire... ce qui est peu. Georges Mabille veut faire comprendre à son lectorat français que la situation est ancienne : "Les gens de couleur sont habitués, depuis leur plus tendre enfance, à cette ségrégation que la législation actuelle ne fait que renforcer en la consacrant. Noirs, métis et indiens, sauf dans de rares exceptions, n'ont jamais été admis dans les églises, les cours de justice ou les hôpitaux des blancs ${ }^{35}$. » Il sous-entend donc probablement que les mentalités sont figées dans leurs stéréotypes et que faire évoluer la situation ne sera pas facile. De fait, il faut attendre le massacre de Sharpeville, le 21 mars 1960, pour que la communauté internationale réagisse à grande échelle.

Pour Georges Mabille, le scandale le plus évident de l'apartheid est que des chrétiens puissent à la fois se définir comme tels et soutenir un système injuste et injustifiable :

Que des politiciens blancs aient inventé toutes sortes de restrictions pour limiter le droit des gens de couleur, Michael pouvait encore l'admettre. Ces Blancs-là n'étaient pas des chrétiens. Mais que des églises [sic] qui se prétendent respectueuses de la Parole de Dieu osent proclamer, comme le faisait l'Église Réformée Hollandaise (Boer), que la Parole de Dieu enseigne la doctrine de l'apartheid - c'est-à-dire de la ségrégation raciale - et le gardiennage des Noirs par les Blancs, cela dépassait son entendement! Dieu n'a-t-Il pas créé le sang des Noirs du même rouge que celui des Blancs ${ }^{36}$ ?

Son expérience sur place lui permet de ne pas généraliser et ainsi de constater tout n'est pas homogène : il a des différences entre les individus, qui ne se comportent pas tous de la même manière. On ne peut donc pas avoir une approche trop simpliste de la situation, même si, sur le fond, le problème reste le même. Il souligne un fait important mis en avant après l'élection de Mandela, devant la commission Vérité et Réconciliation, par les autres Églises souvent dites «anglophones» (presbytérienne, anglicane, méthodiste, baptiste, etc.) mais également par les personnes de confession juive et dans une moindre mesure musulmane : toutes reconnaissent ne pas avoir assez vigoureusement lutté contre l'apartheid, en particulier parce que faire partie d'une élite blanche était très confortable :

Les quelques églises [sic] blanches qui, avec une certaine condescendance, autorisent les Noirs à s'asseoir sur les derniers bancs du temple, ne sont pas appréciées des autres églises [sic] blanches. Les prières dans les églises des Blancs ne sont pas sincères parce qu'il y a un abîme entre la prédication sur la responsabilité qu'en tant qu'enfants de Dieu nous avons les uns pour les autres et le fait qu'en quittant l'église nous acceptons tous les privilèges des Blancs que nous vaut la discrimination et tout le mal fait aux non-Blancs ${ }^{37}$.

Un fait intervenu à l'occasion du mariage de sa fille Claire avec Pierre Casalis en septembre 1952 offre un autre exemple de ségrégation raciale vécu par Georges et sa famille. Claire a une amie noire de son âge qui, en hommage à leur amitié, a décidé de se marier le même jour qu'elle. Mais en dépit du vœu des deux couples et du soutien des parents, les autorités civiles refusent que les deux mariages aient lieu dans le même temple. L'apartheid

\footnotetext{
${ }^{34}$ Ibid.

${ }^{35}$ Ibid.

${ }^{36}$ G. MABILlE, Un Noir + un Blanc $=$ une équipe, op. cit., p. 96.

${ }^{37}$ Ibid., p. 98 .
} 
va jusqu'à ségréguer les lieux de culte. Claire et Pierre, en tenue de mariés, se rendent donc, avec une partie de leurs invités, dans le temple réservé pour les Noirs afin d'assister à la bénédiction de mariage du couple africain, avant d'aller à leur tour se marier dans le temple pour Blancs ${ }^{38}$. Roland Mabille, bien qu'alors âgé de quatre ans, se souvient qu'il était garçon d'honneur et que la petite fille de son âge qui servait de demoiselle d'honneur n'avait pas voulu (ou pu) venir au temple pour les Noirs. Le sentiment de Roland Mabille aujourd'hui est que cela était interdit et que tous les Blancs invités au mariage de Claire et Pierre n'avaient donc pas voulu enfreindre la loi $^{39}$. Pour lui qui vivait dans une famille où les distinctions raciales n'étaient pas de mise (il avait été baptisé par le pasteur noir Joshua Selikane), cela ne posait aucun problème, d'autant qu'il y avait régulièrement des Sotho chez eux, comme le souligne Claire qui ne parlait que le sesotho jusqu'à l'âge de trois ans : " Chez nous il y avait des Noirs qui venaient de l'Église ; on en avait souvent à notre table et papa leur parlait en sesotho $^{40}$. »

C'est en cela que Georges Mabille et sa famille sont des « exceptions normales ${ }^{41}$ » selon la définition de la microhistoire. La séparation entre groupes ethniques est la norme en Afrique du Sud depuis le vote des premières lois de l'apartheid : «Loi sur la prohibition des mariages mixte » (loi $\mathrm{n}^{\circ} 55$ de 1949) qui interdit les mariages entre groupes ethniques différents, et l'amendement de la «Loi sur l'immoralité » (loi n 21 de 1950) - amendement seulement car la loi existe depuis 1927, bien avant l'apartheid - dont le but est d'interdire toutes les relations sexuelles entre Blancs et Noirs. Georges cite l'exemple d'une jeune paroissienne noire de Michael Malefane, âgée de dix-sept ans, employée chez des Blancs et séduite par le fils blanc de la famille âgé de 24 ans. La jeune femme est condamnée à quatre mois de prison ferme alors que le garçon, qui aurait dû être condamné selon les termes de la loi, est relâché ${ }^{42}$. Georges Mabille relate d'autres faits plus graves, sans préciser toutefois les noms, dates et lieux : trois policiers blancs ayant torturé et assassiné huit Noirs ne sont condamnés qu'à sept jours de prison ${ }^{43}$. Les témoignages des enfants de Georges et Marcelle (Louise) Mabille sont précieux dans ce contexte : Claire, Christian et Roland sont des témoins oculaires de ce qu'est l'apartheid au quotidien, vécu par tous les Sud-Africains mais appréhendé différemment selon le groupe d'appartenance raciale des uns et des autres.

Claire Casalis née Mabille, Christian et Roland Mabille sont, par exemple, tous les trois marqués par la vision des grilles qui fermaient les portes et les fenêtres de leur maison de Kensington South (4 Leander Street), tant l'insécurité règne dans ce quartier blanc de la banlieue de Johannesburg, situé à côté de la paroisse de Village Main dans la Région F de la City of Johannesburg Metropolitan Municipality. Si, aujourd'hui, la population de Kensington South est pour près de la moitié composée de Noirs, pour seulement $37 \%$ de Blancs, à l'époque, et avant même que l'apartheid ne soit instauré, les groupes sont séparés. Roland Mabille, né en Afrique du Sud en 1948 et qui y vit jusqu'à l'âge de huit ans, se souvient qu'il ne côtoyait que des Sud-Africains noirs, employés de maison ou bien commerçants indiens (exception faite des visiteurs de ses parents). Les Blancs vivaient dans la peur, explique-t-il avant de l'illustrer par une anecdote. Une nuit, il voit apparaître à sa fenêtre ouverte le visage d'un Noir éclairé de façon diffuse par sa torche, ce qui le rend méconnaissable. Il crie pour appeler ses parents ; or il s'avère que le jeune noir est leur boy,

\footnotetext{
${ }^{38}$ Entretien avec Claire (Mabille) Casalis, Marseille, 25 juin 2019.

${ }^{39}$ Entretien téléphonique avec Roland Mabille, 29 juin 2019.

${ }^{40}$ Entretien avec Claire (Mabille) Casalis, Marseille, 25 juin 2019.

${ }^{41}$ Le concept d' « exception normale » en microhistoire indique qu'une unité d'étude (ici la famille Mabille) est une exception qui confirme la règle sociale qui est d'obéir aux lois de l'apartheid. Cette unité a du sens du point de vue historique, elle est normale car elle incarne un contre-exemple de norme sociale. Voir Carlo GuinZBurg, Carlo PONI, « la micro-histoire », Le Débat 17 (1981), p.133-136.

${ }^{42}$ G. MABILle, Un Noir + un Blanc $=$ une équipe, op. cit., p. 120.

${ }^{43}$ Ibid.
} 
employé de la maison, qui n'avait pas ses clés. Ce qui aurait pu prêter à sourire se révèle, dans ce contexte, anxiogène pour toute la famille Mabille. Un autre jour, Christian et Roland trouvent une couverture coincée dans le grillage de la fenêtre de la chambre de Claire ; il y avait juste une petite trappe pour faire jouer l'ouverture de la vitre et des voleurs ont essayé de dérober la couverture en la tirant par le dehors, mais n'ont pas réussi : la couverture et les draps sont restés coincés dans le grillage. Roland se souvient aussi qu'il n'y avait pas de grillage sur les fenêtres de sa marraine suisse, Marthe Seidl ${ }^{44}$, dont le mari travaille dans les mines d'amiante. Ils habitent West Cliff qui est «upper class $^{45}$ », dit Roland, c'est-à-dire un quartier pour les classes supérieures, dans une énorme mansion (une grande demeure) avec un toit de chaume et une piscine, où travaillent au moins trois domestiques noirs dont une cuisinière et un jardinier. West Cliff fait partie des quartiers protégés qui se trouvent loin des townships alors que les Mabille vivent dans un quartier bourgeois (middle class) du secteur blanc, et qu'à ce titre la sécurité y est moindre. Autre anecdote relatée par Roland : un jour qu'il est assis sur le pas de porte du presbytère, son père le tire violemment à l'intérieur de la maison et le sermonne sur son attitude inconsciente : un enfant qui reste seul sur le pas d'une porte, même dans un quartier blanc, se met dans une situation potentiellement très dangereuse. Autre événement, bien plus grave celui-ci : une nuit vers 1956, sa mère entend, depuis sa chambre, les gémissements d'une personne au-dehors recevant des coups. Elle tente en vain de réveiller son mari profondément endormi. Le lendemain, on découvre en haut de la rue le cadavre d'une femme noire battue à mort. Ce drame hante Georges toute sa vie : "Je me souviens, précise Roland Mabille, mon père disait qu'il en avait gros sur la conscience de ne pas s'être réveillét ${ }^{46}$ »

Ces difficultés rendent la vie de la famille difficile. Claire Mabille raconte que sa mère trouvait les conditions de vie en Afrique du Sud si dures qu'elle déclara un jour que si elle avait su, elle n'aurait pas fait d'enfant ! Des propos confirmés par Pierre Casalis, alors fiancé de Claire, qui se souvient de l'anecdote un sourire aux lèvres : «Elle m'a dit ça à moi qui venait de France pour épouser sa fille ! » Quant à Roland Mabille, il se rappelle avoir entendu sa mère se plaindre que faire un pique-nique au milieu des cactus et des scorpions à Wilgespruit n'est pas une chose agréable, et qu'elle regrette la France et l'herbe verte du Béarn à l'ombre des grands arbres. Cela ne l'empêche toutefois pas d'organiser de belles fêtes paroissiales, ajoute Roland. Outre l'environnement social, une des raisons de ces difficultés tient au fait que Georges Mabille est absorbé par son travail : «Il était là sans l'être ${ }^{47}$ », souligne Claire. Georges Mabille a beaucoup d'activités paroissiales, mais aussi extraprofessionnelles : "Ce qui a beaucoup compté, dit Claire, c'est que Papa a été secrétaire général de l'Alliance Française [à Johannesburg], il accueillait beaucoup de gens. Je ne sais pas comment il a géré tout cela ${ }^{48}$. » Claire précise que c'est une vie que son père a aimée pardessus tout. Se souvenant d'une conversation avec son frère Christian - qui lui rappelait à quel point sa sœur et lui, ainsi que leurs deux plus jeunes frères (Frank et Roland), avaient été élevés à la dure -, elle ajoute que leurs parents «vivaient cela intensément. Nous nous sentions abandonnés car ils n'avaient pas beaucoup de temps ${ }^{49}$. »

Mais si l'insécurité au quotidien, avec ses dangers, est omniprésente, les activités subversives de leur père font planer d'autres menaces au-dessus de leurs têtes. Ainsi Georges Mabille a prévenu ses enfants des risques qu'il encourt: "Je vais à Wilgespruit, si je ne

\footnotetext{
${ }^{44}$ Née Hentsch, de la banque genevoise du même nom.

${ }^{45}$ Entretien avec Roland Mabille, Christian Mabille et Clara Mabille, Carcassonne, le 5 juillet 2019, de 10h à $16 \mathrm{~h}$.

${ }^{46}$ Ibid.

${ }^{47}$ Entretien avec Claire (Mabille) Casalis, Marseille, 25 juin 2019. i

${ }^{48}$ Ibid.

${ }^{49}$ Ibid.
} 
reviens pas, ne vous inquiétez pas, c'est que j'ai été arrêté, mais cela ne doit pas vous contrarier $^{50}$.» Pour eux, l'apartheid est visible tous les jours. Ainsi, Christian passe l'équivalent du baccalauréat à dix-huit ans en Afrique du Sud en décembre 1956 ; il raconte que les enfants Mabille «allaient dans des High Schools anglaises ${ }^{51}$ ». Les écoles ne sont pas mélangées : il y a les écoles anglophones, les écoles pour Afrikaners, mais aussi les écoles pour filles (Jeppe High Schools for girls), « et moi j'allais à Jeppe High School for boys ${ }^{52}$, et donc c'était l'enseignement britannique dans toute sa splendeur: prof de langue, très "Oxford", en robe noire, etc., qui décortiquait Shakespeare avec de grands gestes ${ }^{53} \ldots$ » Dans le cadre scolaire, un entraînement militaire lui est prodigué :

Le jeudi, nous avions tous reçu un uniforme kaki, chemise kaki, t-shirt kaki, ceinturon, et une casquette de l'Afrika Korps (ça, ça m'avait choqué)... c'était le même dessin tu vois. Et le jeudi on allait... on marchait au pas, on apprenait à tirer au fusil, un petit entraînement et dans mon esprit, j'avais entendu que si jamais les Noirs bougeaient... on préparait une espèce de milice. Ça c'était un signe très fort de l'apartheid ${ }^{54}$.

Christian est également marqué par la ségrégation dans les transports : "Les vieux tramways un peu brinquebalants étaient pour les Noirs, ils étaient gris, tristes, c'étaient les mêmes rails tout de même, mais pas les mêmes arrêts et pour l'arrêt des Blancs il y avait écrit for Whites only ${ }^{55}$. » La ségrégation est partout, explique-t-il, sauf dans les commerces (sousentendant ainsi que l'argent n'a pas de couleur), mais aussi les parcs où les nounous sont autorisées à se rendre, mais doivent rester debout si les enfants qu'elles gardent s'assoient sur les bancs for Whites only ou for Europeans only (sauf exception quand elles ont des bancs pour elles, «pour nounous seulement »-for nannies only). Sa sœur Claire se souvient bien des bancs réservés aux Européens (terme désignant les Blancs en général pendant cette période). Pour elle, c'est là le souvenir le plus marquant de ce qu'était l'apartheid : "C'était très fort cette séparation ${ }^{56}$. » On peut sans doute dire que ces bancs ségrégués sont la marque la plus visible de l'apartheid, au point d'avoir envahi la mémoire et l'imaginaire collectifs. À ce sujet, André Brink, le célèbre romancier afrikaner anti-apartheid, aime raconter l'anecdote suivante : le jour où, jeune étudiant sud-africain à Paris dans les années 1960, un Noir vient s'asseoir sur le même banc que lui sans que personne n'en soit ému, il comprend que quelque chose ne va pas dans son pays.

Pour Christian Mabille, les jeunes de son entourage et lui-même n'avaient pas de conscience politique, ils n'étaient pas politisés. Il y a toutefois une conscience d'appartenance à un groupe : ainsi il est traité de froggy par les anglophones ${ }^{57}$. Sa sœur Claire raconte que le mélange entre Blancs n'allait pas de soi : «On supportait mal les Afrikaners, ils avaient un

\footnotetext{
${ }^{50}$ Ibid.

${ }^{51}$ Entretien avec Roland Mabille, Christian Mabille et Clara Mabille, Carcassonne, le 5 juillet 2019, de 10h à $16 \mathrm{~h}$

${ }^{52}$ Ibid. Situé à Kensington, non loin du presbytère où habitent les Mabille à Kensington South, c'est l'un des vingt-trois collèges de mineurs et l'établissement scolaire le plus ancien de Johannesburg, créé par les Anglicans en 1890, puis racheté par la municipalité en 1896.

${ }^{53}$ Ibid (Entretien avec Roland Mabille, Christian Mabille et Clara Mabille, Carcassonne, le 5 juillet 2019, de $10 \mathrm{~h}$ à $16 \mathrm{~h})$

${ }^{54}$ Ibid

55 Ibid

${ }^{56}$ Entretien avec Claire (Mabille) Casalis, Marseille, 25 juin 2019.

57 Froggy vient de frog («grenouille »), terme utilisé par certains Britanniques pour désigner de manière péjorative les Français (mangeurs de cuisses de grenouilles), alors que le terme français pour désigner de manière péjorative les Britanniques est rosbeef.
} 
parti pris, un air supérieur, on le sentait en tant qu'enfant ${ }^{58}$. » Christian se souvient aussi avoir vu quelques arrestations musclées de Noirs. Ces derniers font de la bière cafre (kaffir beer); s'ils zigzaguent sur la voie publique, les policiers tracent une ligne à la craie blanche sur le trottoir et il faut qu'ils la suivent en marchant. S'ils ne marchent pas droit, ils sont emmenés au poste de police ; de même s'ils n'ont pas de pass, c'est-à-dire de pièce d'identité. Christian Mabille se souvient bien de la descente de policiers chez eux, à Kensington, pour aller fouiller la chambre de leur boy, où ils trouvent un bidon avec de la kaffir beer. Roland explique que généralement les Noirs enterraient le bidon pour qu'on ne le trouve pas. Ces souvenirs de la ségrégation raciale en Afrique du Sud, de ses conséquences sociales, émanent de personnes qui sont enfants ou adolescents à l'époque, qui subissent ce qu'ils voient et en gardent une empreinte mémorielle indélébile. Mais chez Georges Mabille, qui est adulte, la vision de l'apartheid embrasse beaucoup plus d'aspects. Il tient des carnets toute sa vie, entretient une correspondance riche et quotidienne. De plus, il a toujours le désir de témoigner dans des conférences, des livres ou des articles.

Témoin privilégié de ce que l'apartheid signifie au quotidien, il n'a de cesse de dénoncer dans ses écrits et conférences la réalité d'un tel système. En 1976, il écrit par exemple :

Tous les visiteurs qui, pour la première fois, mettent le pied en Afrique du Sud sont frappés par la séparation imposée entre Blancs et Noirs. Les trains ont des wagons séparés, les gares des entrées différentes, les bureaux de poste des comptoirs et parfois des salles différentes. Dans les villes, il y a des autobus de couleur différente pour les Blancs et pour les Noirs. Dans les parcs publics, les bancs ont des écriteaux : Européens seulement. Il est impossible pour un homme de couleur de pouvoir consommer dans un restaurant pour Européens, comme il est tout à fait inconcevable pour un Blanc d'aller manger dans un restaurant pour Noirs. À l'époque où Michael [Malefane] vivait sur le Rand, seuls les magasins s'ouvraient aussi bien aux Noirs qu'aux Blancs! Les Noirs travaillaient dans les mines d'or et dans les usines, dans les fermes des Blancs et dans leurs maisons. À part les domestiques, les Noirs ne vivaient pas dans les villes des Blancs. Ils vivaient dans des locations en dehors de la ville et dans les compounds ou campements des mineurs ${ }^{59}$.

L'habitude d'être séparés entraîne des surprises énormes lorsque le mélange se fait malgré tout. Ainsi, lorsque son ami sotho, l'évangéliste Michael Malefane, arrive à Wilgespruit pour la première fois, il « a la surprise d'être accueilli par des inconnus : Blancs, Métis, Indiens et Noirs, évangélistes ou pasteurs, comme lui. Les fraternelles poignées de mains, les grandes tapes amicales dans le dos, les salutations en anglais, en zoulou, en sotho, les grands éclats de rire décontractés et cascadants, tout cela est inconcevable, absolument nouveau ${ }^{60}$. » Un jour, lors d'une session de ce que le pasteur Mabille appelle du «recyclage biblique ${ }^{61}$ », les membres de la congrégation attendent l'évangéliste noir aveugle, Velile, et un pasteur blanc écossais, directeur de l'École biblique de Lovedale en Afrique du Sud, venus tous deux donner les cours. Ils voient alors, de loin, un homme marchant à flanc de colline, portant un autre homme sur son dos : "Quelle ne fut pas la surprise de toute la communauté, constituée en vaste majorité de Noirs, ce matin-là, de réaliser qu'il s'agissait de l'évangéliste aveugle Velile que le missionnaire portait sur son dos! Impensable en Afrique du Sud : un pasteur blanc portant pour un aussi long parcours un évangéliste zoulou ${ }^{62}$. » De même, des Blancs de

\footnotetext{
${ }^{58}$ Entretien avec Roland Mabille, Christian Mabille et Clara Mabille, Carcassonne, le 5 juillet 2019, de 10h à $16 \mathrm{~h}$.

${ }^{59}$ G. MABILLE, Un Noir + un Blanc = une équipe, op. cit., p. 95.

${ }^{60}$ Ibid., p. 109.

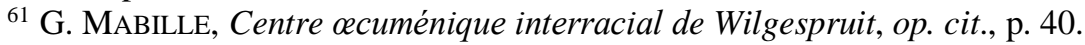

${ }^{62}$ G. MABILlE, Un Noir + un Blanc = une équipe, op. cit., p. 110. Notons que dans ID., Centre occuménique interracial de Wilgespruit, op. cit., p. 40, Mabille dit que Velile est xosa [sic].
} 
passage acceptent de se mettre sous les ordres d'Edwin Ratsatsi, un Noir en charge d'une partie des travaux du Centre ${ }^{63}$. Dans le même ordre d'idée, Michael Malefane devient acteur de ces mélanges interethniques: "Quelles excitantes parties de saute-mouton et d'attrape furent alors organisées par Michael sur l'herbe verte où les partenaires faisaient l'effet de pions de dominos noirs et blancs ${ }^{64}$. » Ce que Georges Mabille souligne constamment dans ses écrits, principe valable pour tous les êtres humains mais qui résonne de manière particulière au pays de l'apartheid, est qu'on ne peut se faire une idée d'une personne que si l'on a pu lui parler, établir une relation de confiance avec elle et ainsi l'écouter parler d'elle pour mieux la découvrir :

Souvent aussi, nous nous contentions de nous asseoir méditant et écoutant ce que chaque campeur avait à nous dire du problème racial et comment il se sentait concerné. C'était très révélateur d'entendre les commentaires de campeurs africains, car il est très difficile de découvrir ce que pensent les Africains, sauf quand on peut établir des contacts personnels avec eux ${ }^{65}$.

Par ailleurs, et c'est aussi en cela qu'il est précieux pour les historiens de la période de l'apartheid en Afrique du Sud, Georges Mabille met en tension ce qui est vécu par lui-même et ses hôtes de Wilgespruit, avec l'Afrique du Sud du dehors, la blanche, celle qui adhère au message du gouvernement, probablement par crainte du Noir qu'elle ne connaît pas :

À plusieurs reprises, avant mon arrivée à Wilgespruit, les automobilistes qui m'offraient une place au cours de mon voyage essayaient de résumer la position des meneurs africains éduqués. On me disait par exemple que le Congrès national africain [ANC] voulait rejeter complètement l'homme blanc hors de l'Afrique ; le vrai responsable chrétien du peuple africain était en faveur de l'apartheid ; l'ouvrier africain ordinaire sur les fermes ou dans l'industrie avait une totale confiance dans la bonne volonté de ses patrons blancs à moins de n'être excité par des agitateurs communistes ou des Kafferboeties (« amis des nègres ») ${ }^{66}$.

Ce qui relève du préjugé chez les Blancs qui ne s'intéressent pas vraiment aux Noirs est un frein à la bonne entente entre les peuples. Georges Mabille et les autres cofondateurs du Centre sont reconnaissants vis-à-vis de ces Noirs qui viennent à Wilgespruit car ils s'exposent eux aussi à l'opprobre des autres Noirs qui, là aussi par méconnaissance, les accusent de pactiser avec l'ennemi. Pourtant, c'est dans ces rencontres que les Blancs du Centre peuvent comprendre que les discours du gouvernement, discours qui se veulent rassurants, ne sont bien souvent que de la propagande destinée à faire croire que l'apartheid est la solution aux problèmes des Blancs :

Ils [les Noirs de Wilgespruit] nous disaient ce qu'ils pensaient de l'Apartheid en tant que chrétiens africains : que c'est une politique de total égoïsme de la part des Blancs et que ce que les Nationalistes prétendent appeler les avantages de l'Apartheid pour les non-Blancs n'est qu'un paquet d'illusions. Ils pouvaient par ailleurs nous montrer que l'Africain moyen sans instruction n'a pas besoin d'un «agitateur communiste» ou d'un «kafferboetie » pour le convaincre qu'il est traité injustement ${ }^{67}$.

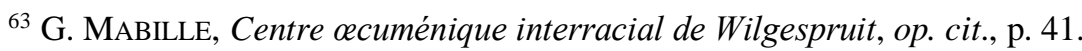

${ }^{64}$ G. MABILlE, Un Noir + un Blanc = une équipe, op. cit., p. 111.

${ }^{65}$ G. MABILLE, Centre 2 cuménique interracial de Wilgespruit, op. cit., p. 48.

${ }^{66}$ Ibid.

${ }^{67}$ Ibid., p. 49.
} 
Cette approche empirique de l'apartheid qui est celle de Georges Mabille se catalyse autour du centre œcuménique (Wilgespruit Fellowship Center) qu'il cofonde en 1948, véritable camp retranché dans sa lutte contre l'iniquité.

\section{WILGESPRUIT, UN LAAGER ANTI-APARTHEID AU SEIN DE L'AFRIQUE DU SUD}

Le laager, en afrikaans, est le camp retranché que les colons boers mettent en place pendant le Grand Trek (1835-1846) en disposant leurs chariots en cercle, et en plaçant des buissons épineux entre chaque chariot pour éviter toute infiltration d'animaux sauvages ou de guerriers bantous hostiles. Ce mot est donc synonyme de défense. Il est associé par les Afrikaners eux-mêmes au système de l'apartheid, qui est pour eux un moyen de se protéger des attaques extérieures ou intérieures. On sait que l'apartheid n'existe officiellement qu'avec l'élection du Parti national de Daniel François Malan, le 26 mai 1948. Néanmoins, les pratiques ségrégationnistes existent depuis longtemps, et les Afrikaners ne sont pas les seuls à promouvoir un tel fonctionnement social; les colons britanniques ont aussi leur part de responsabilité dans la mise en place d'une société ségréguée et la montée vers ce qui deviendra l'apartheid.

Lorsque Georges Mabille arrive à Johannesburg en 1946 avec sa famille, la situation politique a bien changé depuis la période d'avant-guerre. Le nationalisme afrikaner est au plus haut, même si les élections qui se préparent pour 1948 ne laissent rien présager du résultat. Georges Mabille semble prendre rapidement parti, et le regard qu'il porte à la fois sur son œuvre missionnaire et sur les Africains le conduit naturellement à s'opposer à la ségrégation raciale. Dans sa thèse soutenue en 1934 se trouve déjà l'affirmation selon laquelle le missionnaire doit rechercher la vérité de l'existence humaine dans les relations entre personnes : dans cette recherche, l'Africain est le maître, l'Européen le disciple ${ }^{68}$. À la question rhétorique qu'il pose de savoir comment baptiser du signe du Christ la vie de l'Afrique qui change et de l'Afrique à venir, il répond : "Nous le pourrons en adoptant une politique de travail positif et non de démolisseurs. Nous le pourrons en édifiant des personnalités chrétiennes dans l'ambiance de leur héritage racial ${ }^{69}$. $»$ Lorsqu'il évoque une civilisation européenne atomisée par rapport à une société africaine unifiée ${ }^{70}$, il souligne que détruire cette unité est un risque trop criminel pour être accepté et que l'une « des raisons de la graduelle destruction du cadre de la société tribale provient de la politique de répression adoptée par les premiers missionnaires ${ }^{71} »$. Il ajoute aussitôt que les missionnaires ne sont pas les seuls responsables, et sa condamnation de l'attitude coloniale caractéristique de sa propre société, européenne, est ferme :

La civilisation occidentale est la grande coupable et la disparition des vieilles coutumes indigènes est consécutive à l'introduction des mœurs européennes en Afrique. Cette désintégration de l'ordre social a causé de graves préjudices à la société indigène et le mal en est arrivé à un tel degré de profondeur et d'acuité qu'il est aujourd'hui trop tard pour l'enrayer. Le relâchement des liens organiques de la société indigène est un fait accompli ${ }^{72}$.

Cette citation fait écho aux aberrations de l'apartheid qui détruisent la société africaine pour mieux la gouverner. On note de fortes similitudes entre ce constat de Georges Mabille

\footnotetext{
${ }^{68}$ G. MABILLE, Les écoles d'initiation en Afrique, op. cit., p. 166.

69 Ibid.

${ }^{70}$ Ibid., p. 165.

${ }^{71}$ Ibid., p. 5.

72 Ibid.
} 
datant de 1934 et la citation suivante à propos de l'apartheid rédigée quarante-deux ans plus tard :

Est-il juste que les Blancs dominent jusque dans les églises édifiées parmi les Noirs et pour les Noirs ? Certes ce sont les Blancs qui ont apporté l'Évangile à l'Afrique et qui l'ont fait bénéficier de la civilisation occidentale, mais n'est-ce pas cette civilisation qui a jeté à bas et détruit les fondations de la société indigène? Les institutions traditionnelles bantoues qui sauvegardaient la dignité et l'ordre au sein des peuples d'Afrique australe se sont maintenant effondrées ${ }^{73}$.

Georges Mabille examine avec finesse la situation de l'Afrique du Sud, et son analyse rejoint celle du pasteur et théologien néerlandais Willem A. Visser't Hooft (1900-1985), venu en Afrique du Sud en 1952 en sa qualité de secrétaire général du Conseil œcuménique des Églises. Deux ans plus tard, ce dernier écrit qu'un des trois piliers des difficultés raciales en Afrique du Sud est la présence de nombreux Européens parmi des populations africaines encore plus nombreuses, la présence de nombreux Asiatiques ainsi que «les effets particulièrement dévastateurs de la civilisation occidentale moderne et de l'industrialisation sur la vieille société tribale, rurale et collectiviste, dans cette région où les Européens sont nombreux ${ }^{74} »$.

Georges Mabille n'en oublie pas pour autant les Blancs sud-africains. Il souligne ainsi les enjeux de l'apartheid pour les Afrikaners, enjeux au sujet desquels il échange avec ses amis, le célèbre écrivain Alan Paton et l'évangéliste Michael Malefane: «D'accord avec Paton, Michael estimait que la question raciale était une question de justice ou d'injustice, d'humanité ou d'inhumanité alors que pour les tenants de l'apartheid elle est une question de vie ou de mort ${ }^{75}$. » Mais c'est au quotidien que l'apartheid se décline : "Pour un Afrikaner moyen, toucher la main d'un Noir, boire une tasse de thé avec lui, le traiter de "Monsieur", c'est se rabaisser. Il en découle que partager la chambre d'un Noir et travailler à son niveau, avec lui, c'est un véritable scandale ${ }^{76}$. » Georges Mabille est amené à prendre des décisions qui relèvent de la lutte anti-apartheid; d'ailleurs, le 13 octobre 1954, le directeur de la Mission de Paris, le pasteur Charles Bonzon, lui écrit pour lui confirmer que la décision qu'il a initiée, prise par la Commission exécutive du Lessouto, de rompre les accords passés autrefois par le pasteur-missionnaire François Coillard (1834-1904) avec la NGK, est acceptée par la Mission de Paris ${ }^{77}$. La manifestation la plus probante de l'opposition de Georges Mabille à l'apartheid est la création du Wilgespruit Fellowship Center (WFC).

Le centre de Wilgespruit (littéralement « source des saules ») est une épine dans le pied du gouvernement de Malan, une sorte de camp retranché multiracial au sein de l'apartheid. Comme nous l'avons mentionné, il est cofondé par Georges Mabille et cinq autres personnes : Jacob Nhlapo (1903-1957), Joshua Selikane, Dante Anderson, Arthur Blaxall (1891-1970), Darrell Randall (1916-2008). Tous d'origines différentes (zoulou, sotho, sud-africaine anglophone, anglaise, américaine, française) mais tous hommes d'Église ou membres d'Églises protestantes (méthodiste, anglicane, congrégationaliste, réformée); tous sont dits daltoniens au sens anglais de colour blind, c'est-à-dire, littéralement, «aveugles aux couleurs » (et donc ne distinguant pas les Noirs des Blancs) ${ }^{78}$. Ce qui, en revanche, les différencie des Sud-Africains pro-apartheid, selon Georges Mabille, est leur foi commune en

\footnotetext{
${ }^{73}$ G. MABILLE, Un Noir + un Blanc = une équipe, op. cit., p. 97.

${ }^{74}$ Willem A. VisSER'T HoOfT, Le mouvement æecuménique et la question raciale, Paris, Unesco, 1954, p. 2930.

75 Ibid., p. 99.

${ }^{76}$ G. MABILlE, Centre œeuménique interracial de Wilgespruit, op. cit., p. 9.

${ }^{77}$ Fonds Mabille, Défap.

${ }^{78}$ G. MABILLE, Centre 2 cuménique interracial de Wilgespruit, op. cit., p. 17.
} 
Jésus-Christ: "Pour nous, il était impensable d'envisager que le chrétien puisse être raciste $^{79}$. » Dans un texte manuscrit peut-être écrit vers 1965, Georges Mabille explique la motivation des fondateurs de Wilgespruit presque vingt ans plus tôt : «En Afrique du Sud l'expérience tentée cherche à découvrir le commun dénominateur de vie chrétienne qui transcende toutes les formes conventionnelles et toutes les habitudes qui séparent l'homme de l'homme $^{80}$. » Il est intéressant de noter, à la lecture de son récit sur les prémices de l'apartheid, qu'il s'agit d'une période d'hésitation, de recherche et de réflexion. L'apartheid est une telle nouveauté que peu de gens ont réfléchi à ce que cela implique et la position de chacun dépend de nombreux facteurs :

En 1947, le gouvernement Smuts s'essoufflait, et nous étions conscients que des élections générales risquaient d'amener au pouvoir les nationalistes du Dr Malan. Les Églises se cherchaient sur le plan des relations raciales [...]. Des conférences groupaient l'Église boer [la NGK], et les Églises du Conseil chrétien. Nous portions le poids d'une situation faite de préjugés raciaux et du désir de se donner une bonne conscience. Mes amis Blaxall, Anderson, Nhlapo, Randall et moi, nous [nous] retrouvions dans les coulisses d'Adams College ou de Pretoria [...].

Nous commentions le rapport de Reyneke, puis celui de Keet et bientôt le livre d'Alan Paton «Pleure, ô mon pays bien-aimé » allait paraître [1948]. Avec Joshua Selikane, j'allais retrouver mes quatre amis dans l'École que dirigeait Nhlapo, situé à l'Ouest de Krugerdorp. Nous formions l'embryon d'une Communauté de Prière et de Réflexion ${ }^{81}$.

Situé au Nord-Ouest de Johannesburg, dans la direction de Krugersdorp et à moins de quatre kilomètres de Roodepoort, Wilgespruit se trouve à côté des deux instituts créés par Arthur et Florence Blaxall, l'un pour les aveugles, Ezenzeleni («Là où tu te prends en charge » en zoulou), et l'autre pour les sourds, Kutlawanong (« Là où l'on entend » en sesotho). La raison d'être de ce centre est indéniablement de s'opposer au cœur de l'idéologie de l'apartheid : il s'agit de montrer que Noirs et Blancs sont égaux devant Dieu, contrairement à ce que le gouvernement de Malan tente pourtant de faire croire. C'est ce qu'exprime Georges Mabille lorsqu'il évoque les débuts du centre : «En fait, les occasions de rencontre entre les représentants des églises [sic] et des races différentes causeront de plus en plus de problèmes ${ }^{82}$. »

Le point souvent omis par les auteurs d'articles universitaires sur Wilgespruit est l'aspect confessionnel de la démarche des six fondateurs, rarement mentionnés comme pasteurs ou ministres protestants. Georges Mabille est fier de souligner ce qui unit les fondateurs :

Invités chez notre ami zoulou en 1947, celui-ci, Jacob Nhlapo était directeur d'une grande école méthodiste et nous étions cinq, quatre blancs et un noir, pasteurs. Nous nous sommes engagés ensemble à créer une communauté de prière dans laquelle nous représentions nos confessions anglicane, réformée, méthodiste, évangélique du Lesotho et congrégationaliste. Blaxall était très intéressé par le mouvement des Ashrams en cours de création en Inde. Mais celui-ci devait dépendre d'un groupe de personnes susceptibles de s'engager à plein temps. Or aucun de nous n'était disponible. Nous avions cependant décidé de nous mettre à la recherche d'une propriété pouvant devenir un centre de retraite pour y organiser des camps de jeunesse ou des sessions de conférences interraciaux ${ }^{83}$.

\footnotetext{
${ }^{79}$ Ibid.

${ }^{80}$ G. MABILLE, «Une expérience communautaire en Afrique du Sud », art. cit., p. 51.

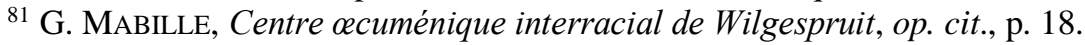

${ }^{82}$ G. MABILlE, Arthur Blaxall, victime de l'Apartheid, op. cit., p. 2.

${ }^{83}$ Ibid., p. 2 sq.
} 
La ferme de vingt-quatre hectares d'un voisin du centre pour aveugles est achetée et nommée Centre œcuménique interracial de Wilgespruit (en anglais Wilgespruit Fellowship Centre), du nom d'une des premières mines d'or du Witwatersrand qui se trouve dans cette vallée. La direction en est confiée au Conseil des Églises qui met un an avant d'accepter car, comme le fait remarquer Georges Mabille, «sous le régime de l'apartheid, [cela] pouvait être un cadeau empoisonné ${ }^{84} »$.

Roland Mabille raconte qu'il est à l'époque trop jeune pour faire des travaux de manutention à Wilgespruit, en chantier permanent, mais que son frère aîné Christian-peine beaucoup sur les divers chantiers qui se succèdent. Toutes les bonnes volontés sont les bienvenues pour construire le Centre. De plus, cela fait partie de la règle de Wilgespruit : celles et ceux qui y viennent, même pour une simple retraite d'un week-end, s'engagent à aider à sa construction et à son entretien. Georges y va régulièrement avec ses enfants lorsqu'ils n'ont pas classe et les met à contribution. Christian Mabille évoque les premiers mois du Centre. Il se souvient de la convivialité et des prières. Il n'a qu'un mois de vacances, et il le passe avec son frère Frank à travailler au WFC : " On construisait les murs du dam [« retenue d'eau » en anglais], on réparait les maisons, c'étaient les camps de travail. » Pour Christian, c'était un monde à part. "À Wilgespruit on a vécu des interdits, on se sentait ailleurs, dans un lieu improbable. Je me souviens de bonnes rigolades entre Blancs et Noirs. » Claire Casalis, confirme l'impression de son frère à propos de Wilgespruit : "C'était merveilleux comme endroit, loin de la ville, il y avait une vallée, un ruisseau, tout le monde partageait tout, c'était hors du temps. » Les activités du Centre, outre « sa propre vie de prière et de communion fraternelle ${ }^{85} »$, consistent en des sessions bibliques (organisées pour les pasteurs africains par l'Association missionnaire du Transvaal), des groupes de discussion, des retraites spirituelles et des camps. Les locaux sont à la disposition des écoles du dimanche, et le vaste domaine peut servir pour les associations désireuses de faire des piqueniques. Pour Georges Mabille, le point d'orgue des activités du Centre est un camp annuel au mois de juillet (hiver austral). Celui-ci peut durer jusqu'à cinq semaines, sur le modèle des camps œcuméniques qui bourgeonnent un peu partout à l'époque dans le monde, et qui réunissent, chaque année, de plus en plus de monde. Les hôtes réguliers du Centre pendant les cinq premières années sont des luthériens, l'Association de la jeunesse anglicane, la Fédération des étudiants chrétiens, l'Armée du Salut, et d'autres encore ${ }^{86}$.

Le redécoupage administratif en zones pour Blancs ou Noirs, que le gouvernement d'apartheid met en place le 27 avril 1950 (Group Areas Act $n^{\circ} 41$ ) perturbe la vie de cette communauté, puisque la zone devient réservée aux Blancs. Les deux centres des Blaxall pour aveugles et pour sourds - doivent déménager à Hammanskraal à 124 kilomètres de là, en zone réservée aux Noirs, au nord de Pretoria. En ce qui concerne le Centre œcuménique, Georges Mabille explique :

Comme notre Centre avait vocation de recevoir des gens de couleur au même titre que les Blancs, nous fûmes l'objet de pressions et de persécutions pour nous forcer à vider les lieux. Par la grâce de Dieu nous tiendrons ferme, face aux menaces et au bout de cinq ans nous avons pu régler nos dettes et enregistrer légalement le Centre ${ }^{87}$.

Georges Mabille jette un voile pudique sur les exactions des forces de police de l'apartheid, mais les méthodes sont connues et souvent efficaces ; la résistance de Wilgespruit n'en est que plus remarquable. Roland Mabille explique qu'il y avait régulièrement des

\footnotetext{
${ }^{84}$ Ibid., p. 3.

${ }^{85}$ G. MABILLE, Centre oecuménique interracial de Wilgespruit, op. cit., p. 37.

${ }^{86} \mathrm{Ibid}$.

${ }^{87}$ Ibid.
} 
descentes de police visant le Centre, ce que confirme son père dans ses écrits : «Le Conseil des Églises a fait de la résistance passive jusqu'au jour où la police, accompagnée de délégués des Affaires bantoues, a fait une descente spectaculaire sur le Centre. Ils ont passé au peigne fin les documents dont étaient porteurs tous les membres noirs présents à Wilgespruit et les ont menacés ${ }^{88}$. » Or, il semble que la plupart de ces visites de la police accompagnée de membres du Département d'administration bantoue se soient déroulées après le massacre de Sharpeville en 1960, comme c'est le cas en 1963, ce qui témoigne d'un contrôle social du gouvernement d'apartheid accru après le massacre. Notons toutefois que le fait que ce soit le fameux Conseil des Églises d'Afrique du Sud, le SACC (South African Council of Churches), qui parraine le centre contribue peut-être à ce que les actions policières ne soient pas trop violentes ni visibles. De même, le fait que les pasteurs fondateurs blancs soient, pour certains, des étrangers (Britannique, Américain et Français) est peut-être un frein aux attaques policières. Néanmoins, tant que dure l'apartheid, les dirigeants du Centre font l'objet de harcèlement et d'intimidations de la part du gouvernement ${ }^{89}$. Par exemple, Arthur Blaxall, directeur du Centre, est la cible des autorités, au point d'être arrêté en 1956 alors qu'il doit se rendre, avec Georges Mabille et leur ami Alan Paton, à une conférence d'Églises protestantes en janvier 1957. La capacité de résistance de son ami Blaxall fait l'admiration de Georges qui lui rend hommage dans un livret qu'il lui consacre en $1988^{90}$. En 1951, dans son rapport annuel à la Mission de Paris, Georges Mabille est bien conscient des dangers, utilisant la rhétorique des missionnaires-martyrs que les lecteurs du Journal des missions connaissent bien :

Nous vous demandons à vous tous, frères et sœurs chrétiens de nous porter devant le trône de toutes Grâces par vos prières. Nous sommes engagés dans un combat immense et notre témoignage est parfois lourd à porter dans un cadre qui est si hostile à notre idéal de vraie fraternité chrétienne. Dieu nous aide à rester fidèles jusqu'au bout; même si nous devons souffrir la persécution ${ }^{91}$.

Toutefois, de l'avis même de Roland Mabille, ce qui cause des problèmes à son père n'est pas tant le gouvernement d'apartheid que le fait qu'à l'intérieur même de l'Église évangélique du Lesotho les choses parfois se passent mal. Roland Mabille se rappelle que son père dut se présenter devant un conseil ecclésiastique pour répondre de charges dont il ne se souvient pas, mais pour lesquelles il fut innocenté. On lui aurait dit : «Tu as fauté, mon frère, mais c'est une sainte faute. » Peut-être avait-il dépassé sa marge de manœuvre, mais pour une bonne raison (du moins selon lui) ? Claire, Christian et Roland Mabille signalent que parfois leur père était dur, envers lui-même, envers ses enfants et ses paroissiens, ce qui explique peut-être cette convocation au conseil de l'Église au Lesotho ${ }^{92}$. Un historien dit à propos de Georges

${ }^{88}$ Ibid., p. 64.

${ }^{89}$ C'est le cas par exemple de Dale White (1934-2007) qui est un prêtre anglican célèbre pour avoir été présent lorsque les enfants de sa paroisse (St Paul Jabavu, White City) se sont révoltés à Soweto en 1976. Ordonné prêtre dans l'Église anglicane à 22 ans en 1956, il est en charge de paroisses métisses ou noires. Il devient le directeur du Wilgespruit Fellowship Center en 1965 (jusqu'en 1993). Ami de Steve Biko, activiste noir anti-apartheid assassiné par la police en 1977, il connaît de nombreux séjours en prison. Son passeport ainsi que celui de sa famille sont confisqués dans les années 1970 et rendus quinze ans plus tard. En avril 2007, peu de temps avant sa mort, il est décoré, par le président Thabo Mbeki, de l'ordre du Baobab pour services rendus à la démocratie.

${ }^{90}$ Voir supra n. 26.

${ }^{91}$ Georges MABILLE, «Le Centre chrétien de Wilgespruit », Journal des missions évangéliques 128 (1953), p. 141-142 (citation p. 142).

${ }^{92}$ Entretien avec Roland Mabille, Christian Mabille et Clara Mabille, Carcassonne, le 5 juillet 2019, de 10h à $16 \mathrm{~h}$ 
«qu'il n'avait pas froid aux yeux ${ }^{93} »$. Christian Mabille insiste sur l'importance de l'éducation dans leur famille, soulignant que leur mère venait d'une famille d'artistes dans laquelle on pouvait prendre le temps de s'amuser, alors que du côté de leur père se manifestait une certaine rigidité : il était strict et exigeant, voire parfois autoritaire; il lui arrivait ainsi de prendre ses fils par le haut du bras, en serrant un peu pour les amener là où il voulait (au sens physique et symbolique du geste). Christian raconte:

Il y avait cette rigueur et cette rigidité protestantes. Je me souviens de cela. Un exemple, une fois : j'étais à la maison à Kensington South sur les figuiers, près de la cuisine, avec un copain on mangeait des figues. Puis Georges rentre d'une tournée ou autre chose, puis il me dit: «Viens ici dans la salle de bain, tends la langue. » Il m'a lavé la langue avec du savon parce que j'aurais menti à ma mère... donc cela m'a marqué aussi ${ }^{94}$.

Christian, lors de notre entretien, exprime le sentiment que son père éprouvait de la gêne : le monde bougeait autour de lui, et sa rigueur pouvait être mal perçue. Comme toujours, il faut situer les choses dans leur contexte. Â la décharge du pasteur Mabille, on peut dire que la responsabilité de missionnaire est lourde, et l'engagement dans une activité missionnaire auprès des Noirs sud-africains au sein de l'apartheid n'a rien d'une sinécure ! Le missionnaire doit aussi savoir tout faire, jusqu'à arracher les dents de ses paroissiens pendant ses tournées afin qu'ils ne soient pas «mangés par leurs dents », comme on le disait au Lesotho en cas de problèmes dentaires !

La question que la microhistoire pose est de savoir ce que l'étude d'une unité (ici, le pasteur Mabille et sa famille) peut apporter à l'histoire globale. L'une des réponses possibles est suggérée par l'historien finlandais Matti Peltonen lorsqu'il écrit qu'un « détail étrange peut représenter un total plus large ${ }^{95} »$. Autrement dit, l'étrangeté de l'attitude de Georges Mabille (et de ses amis de Wilgespruit) qui ne se conforme pas aux lois de son pays d'accueil, l'Afrique du Sud, peut être perçue comme un élément métonymique de la constellation, minoritaire certes, mais réelle, des opposants au régime d'apartheid. Autrement dit, si sur le plan microhistorique Georges Mabille n'est qu'un cas particulier dans le cadre de l'Afrique du Sud du temps de l'apartheid, sur le plan macrohistorique il n'en fait pas moins partie de l'ensemble de tous les êtres humains qui ont uni leurs forces pour déstabiliser et-in fine abattre ce régime.

Dans ce cadre, on peut également faire des observations sur le cas microhistorique de Georges Mabille, afin d'apporter des réponses, sur un plan macrohistorique, à la question posée au sein de la Mission de Paris depuis le début de la mission au Lesotho : les pasteursmissionnaires doivent-ils faire de la politique Lorsqu'il assiste au jubilé de la mission du Lesotho à Morija, le 24 avril 1883, le pasteur Alfred Boegner, directeur de la Mission de Paris, répond en partie à la question. Ce jour-là, il écoute, admiratif, Adolphe Mabille (gendre d'Eugène Casalis) qui essaye avec éloquence de convaincre les Sotho de rester sous la protection de la Couronne britannique. Cette scène inspire à Alfred Boegner la réflexion suivante dans un compte rendu cité par l'historien Jean-François Zorn : «Qu'on dise ce qu'on voudra contre les missionnaires qui font de la politique; je dis que dans ce cas faire de la

\footnotetext{
${ }^{93}$ F. FABRE, Protestantisme et colonisation, op. cit., p. 145.

${ }^{94}$ Entretien avec Roland Mabille, Christian Mabille et Clara Mabille, Carcassonne, le 5 juillet 2019, de 10h à $16 \mathrm{~h}$

95 Matti Peltonen, «Clues, Margins, and Monads. The Micro-Macro Link in Historical Research », History and Theory 40 (2001), p. 347-359 (citation p. 349).
} 
politique c'était faire de la mission au sens le plus élevé du mot ${ }^{96}$. » Boegner souligne ensuite que ce jour-là Adolphe Mabille est pâle et visiblement ému, il s'est exprimé avec force, et l'image qui lui reste est celle d'un messager de la miséricorde céleste ayant pour mission de prévenir encore une fois ce peuple tant aimé.

Ces paroles concernant son grand-père peuvent sans nul doute s'appliquer à Georges Mabille qui a fait de la mission au sens le plus élevé du mot en se rangeant du côté de ses frères sotho, mais aussi de toutes les victimes de l'apartheid. Frédéric Fabre souligne que dans les premiers temps la Mission de Paris est timorée par rapport à l'apartheid. Il est vrai que critiquer les protestants de la NGK, qui pour certains descendent des Huguenots exilés dans les Provinces-Unies (actuels Pays-Bas) et l'Afrique du Sud après la révocation de l'Édit de Nantes en 1685, risque de se révéler contre-productif. De même, s'opposer au gouvernement d'apartheid qui est officieusement dans le camp du bloc de l'Ouest pendant la guerre froide signifie aider les communistes qui soutiennent les indépendances et le combat pour la liberté des Sud-Africains noirs, mené par l'ANC. Il faut attendre 1977 pour qu'enfin le Journal des missions, bulletin officiel de la Mission de Paris, commence à critiquer l'apartheid de manière plus marquée, en dénonçant en particulier les conditions déplorables de vie et de travail des mineurs noirs ${ }^{97}$. Frédéric Fabre accorde cependant une place particulière à Georges Mabille : ce dernier a lutté plus farouchement contre l'apartheid, parce que « lui était sur le terrain ${ }^{98}$ ». Fabre le désigne comme un héros/héraut en concluant sans ambiguïté son chapitre sur l'attitude réservée de la Mission de Paris : «Mais des réactions étaient déjà venues depuis longtemps d'un homme au caractère bien trempé, Georges Mabille ${ }^{99}$. » On le voit, ce dernier est distingué des autres missionnaires en Afrique du Sud.

Olivier Raoul-Duval, actuellement pasteur de l'Église protestante unie de France, se souvient de Georges Mabille, qui fut son paroissien dans l'Église réformée du Coutach (Sauve-Quissac dans le Gard) ${ }^{100}$ et dont-il présida, le 5 décembre 1998 au temple de Sauve, le culte d'action de grâce célébré suite à son décès. Il le décrit comme « un homme passionné et passionnant jusqu'au bout ${ }^{101} \gg$. Claire Casalis se rappelle, quant à elle, que dans les derniers mois de sa vie, son père était tellement impliqué par sa foi dans sa mission au Lesotho et à Johannesburg, et, peut-on sans doute dire, imprégné de son passé, qu'il ne parlait plus que sesotho. Ses enfants avaient autrefois parlé cette langue mais l'avaient en partie oubliée, aussi lui demandaient-ils de parler français. Le pasteur Raoul-Duval se souvient parfaitement que Georges Mabille avait visiblement du plaisir à parler en sesotho avec un autre de ses paroissiens, issu lui aussi d'une grande famille de pasteurs-missionnaires au Lesotho, Paul Ellenberger (1919-2016) ${ }^{102}$. Christian Mabille le confirme, qui se rappelle les avoir entendus

\footnotetext{
96 Alfred BoEGNER, «Quatrième lettre du directeur : les stations de Bethesda, de Siloe, de Thabana-Morena, le Pitso du 24 avril 1883, Bloemfontein, le 12 mai 1883 », Journal des missions évangéliques (1883), p. 242257, cité par Jean-François ZoRN, Le grand siècle d'une mission protestante. La Mission de Paris de 1822 à 1914, Paris, Karthala, 2012, p. 424.

${ }^{97}$ F. FABRE, Protestantisme et colonisation, op. cit., p. 145.

${ }^{98}$ Ibid., p. 152.

99 Ibid.

100 À présent Église protestante unie de Coutach.

101 Échange épistolaire avec le pasteur Olivier Raoul-Duval, 3 février 2015.

102 Entretien avec le pasteur Olivier Raoul-Duval, 24 juin 2019. Notons que ces dynasties de pasteursmissionnaires du Lesotho, comme les Ellenberger, les Casalis et les Mabille, se connaissent bien. À titre d'exemple, rappelons qu'Adolphe Mabille épouse Adèle Casalis en 1859 et que Claire Mabille fait de même avec Pierre Casalis en 1952 ; alors qu'Adolphe Mabille s'installe à Morija en 1860, que Frédéric Ellenberger arrive au Lesotho en 1861 et s'installe à trente kilomètres de là, à Thabana-Morena, ou encore que Louis Mabille succède à René Ellenberger à la mission du Rand, etc. Voir Jean-François ZoRN, « Du Béarn à l'Afrique. Les Casalis, une tribu missionnaire protestante au XIX-XX ${ }^{\mathrm{e}}$ siècles », in Jean-Marie Bouron, Bernard SALVAING (dir.), Les missionnaires. Entre identités individuelles et loyautés collectives (XIX-XX siècles), Paris, Karthala, 2016, p. 91-117.
} 
après un bon repas entre amis dans l'auberge de Sauve qu'il tenait. De retour en France, Georges suivait l'actualité sud-africaine de près, il « se sentait concerné par ce qui s'y passait, il était dedans ${ }^{103} »$, se souvient encore Claire. Elle ajoute :

Et justement en cette fin de vie il était tellement de retour là-bas tout en étant à Sauve qu'il parlait sesotho avec [Paul] Ellenberger. Je me souviens : il y avait Olivier Raoul-Duval, et papa raconte à un moment donné comment se passe un mariage là-bas au Lesotho : " Eh bien, si tu veux une femme il te faut du bétail pour payer», puis comme il commençait à raconter n'importe quoi, il dit à Olivier : «Pour pouvoir épouser ma femme, j'ai dû fournir cinquante bœufs $»^{104}$.

L'Afrique du Sud, «cela passait avant tout, je crois », dit Claire Casalis, au point qu'en février 1990, lorsque Mandela est libéré, des voisins lui racontent que Georges a parcouru toutes les rues de Sauve en criant : «Il est libre, il est libre !» Le petit-fils de Georges Mabille et son épouse, Yves et Isabelle Casalis, regrettent de ne pas avoir pu être les interlocuteurs que Georges aurait peut-être souhaité avoir car, s'ils l'écoutaient attentivement, ils ne pouvaient pas appréhender pleinement le vécu extraordinaire de leur grand-père ni le partager avec lui. C'est aussi ce que confirment les fils de Georges Mabille, Christian et Roland. Yves Casalis explique : "Parfois en famille il avait du mal à partager ça. On sentait bien que ce qu'il avait vécu c'était très fort, en dehors même de l'apartheid ${ }^{105}$. » Isabelle Casalis ajoute :

Je dirais même que ce qu'il a vécu était tellement spécial que cela pouvait le rendre asocial, même avec les proches. Nous étions dans nos vies parisiennes, le métro, les études des enfants, c'est un autre monde qu'il a vécu. Lui était baigné dans un autre monde, et seuls les gens de làbas, sa fille et ses enfants pouvaient le comprendre. Mais nous, nous ne pouvions pas toujours le suivre, ce qu'il ne pouvait pas toujours comprendre. Pour lui, nous n'étions pas un vis-à-vis formidable ${ }^{106}$.

Un proverbe sotho dit que celui qui creuse une source n'en boit pas l'eau. Georges Mabille fait partie de ces personnes qui ont œuvré efficacement contre un système politique et ont planté les graines qui ont contribué à son démantèlement mais dont l'histoire a quelque peu oublié le rôle. Ses actions ne font pas la une des journaux. Il n'est pas arrêté par la police de l'apartheid et ne devient donc pas une icône de la lutte anti-apartheid, mais il est un parfait exemple de la manière dont des individus, portés par leur foi, œuvrent, à leur niveau, contre un système qu'ils jugent inique - petit ruisseau qui devient océan. Comme le souligne Frédéric Fabre : «[Georges Mabille] se bat de toutes ses forces contre l'apartheid, mais sans que son action soit subversive ${ }^{107}$. » À la manière de cet ouvrier allemand, August Landmesser (1910-1944), qui croise les bras sur une célèbre photo de 1936, alors que des centaines de personnes font le salut nazi tout autour de lui, Georges Mabille est de ceux qui ont croisé les bras de la résistance passive au sein même de l'Afrique du Sud de l'apartheid, en cohérence avec leurs convictions, leur foi et le regard bienveillant qu'ils portent sur les êtres humains. Il insuffle une vision du monde différente de celle des idéologues de l'apartheid. Il est une autre voix , n'ayant de cesse d'être témoin du Christ en relatant inlassablement son expérience au travers de ses livres, de ses articles, de ses conférences, mais aussi des voyages qu'il organise pour emmener ses paroissiens en Afrique du Sud. Il commente l'évolution du pays jusqu'à la fin de l'apartheid et l'élection de Nelson Mandela en 1994, lui qui l'a rencontré près d'un

\footnotetext{
${ }^{103}$ Entretien avec Claire Casalis, née Mabille, et sa famille, Marseille, 25 juin 2019.

104 Ibid.

105 Yves Casalis, ibid.

${ }^{106}$ Isabelle Casalis, ibid.

${ }^{107}$ F. FABRE, Protestantisme et colonisation, op. cit., p. 147.
} 
demi-siècle plus tôt. Ainsi prend fin le long trek de Georges Mabille, de Morija au Lesotho jusqu'à Sauve dans le Gard, celui d'un témoin, passeur et acteur d'une époque entrée dans l'histoire, celle des pasteurs-missionnaires de la Mission de Paris, dont l'engagement a parfois dépassé le cadre fixé. Georges Mabille s'est investi dans la défense des Sotho et de la majorité noire en Afrique du Sud. De tout cela, il faut à présent garder trace, comme d'un élément de cette microhistoire qui étudie le comportement et l'attitude de ceux qui ne suivent pas le chemin commun de leur époque. Comme l'indique le sous-titre de son livre sur son ami Michael Malefane, il fait lui-même partie de «ceux qui ont fait tomber la barrière de couleur au pays de l'apartheid ${ }^{108} »$. 